\title{
Comparison of heavy episodic drinking patterns between Korean and Chinese immigrants
}

\author{
Danielle E. Ruderman ${ }^{1}$, John D. Clapp ${ }^{1}$, C. Richard Hofstetter ${ }^{2}$, Veronica $\operatorname{Irvin}^{3}$, Sunny Kang ${ }^{4}$, and Melbourne F. \\ Hovell $^{5}$ \\ ${ }^{1}$ College of Social Work, The Ohio State University, Columbus, Ohio, United States \\ ${ }^{2}$ Department of Political Science and Graduate School of Public Health, San Diego State University, San Diego, California, United States \\ ${ }^{3}$ School of Social and Behavioral Health Sciences, Oregon State University, Corvallis, Oregon, United States \\ ${ }^{4}$ Center for Behavioral Epidemiology and Community Health, San Diego State University, San Diego, California, United States \\ ${ }^{5}$ Center for Behavioral Epidemiology and Community Health, Graduate School of Public Health, San Diego State University, San Diego, \\ California, United States
}

\begin{abstract}
Objective: Drinking-related problems are increasing among Asian immigrants despite the popular perceptions of a "model minority." Sociocultural factors may relate to differing drinking patterns among subsets of Asian American populations. This study explores the relationship between nationality and alcohol consumption among Chinese and Korean Americans, specifically in regards to acculturation and social networks.

Method: First-generation Chinese and Korean immigrants residing in the greater Los Angeles area were recruited $(N=2715)$. Structured interviews were conducted over the phone and by professional bilingual interviewers in the language of participant preference.

Results: Although subsamples were demographically similar, Chinese immigrants were less likely to report heavy episodic drinking (HED) than Korean immigrants. Participants in each group with social networks composed of drinkers or problem drinkers and those that encouraged drinking were more likely to report HED themselves.

Conclusions: Alcohol consumption and its dynamics are impacted by peer networks among first-generation Chinese and Koreans residing in the United States. While drinking behaviors differ for Chinese and Korean immigrants, the impact of peer's drinking behaviors on one's own drinking is paramount. This result has important implications for interventions and the need for further research focusing on the impact of peer interactions and alcohol use among this population.
\end{abstract}

Chinese and Korean Americans are among the fastest growing immigrant populations in the United States, with increases of approximately 43\% from 2000 to 2010 (Hoeffel, Rastogi, Kim, \& Shahid, 2012; U.S. Census Bureau, 2012). Asians/Native Hawaiians/Pacific Islanders report significantly less heavy episodic drinking (HED) than non-Hispanic Whites (Centers for Disease Control and Prevention, 2013). In data collected by the Substance Abuse and Mental Health Services Administration (2010), Asian Americans report the lowest HED rates among various ethnicities, as well as the lowest level of pastmonth alcohol use.

Although Asian Americans typically report lower levels of alcohol consumption than Caucasians (Akutsu, Sue, Zane, \& Nakamura, 1989; Luczak, Wall, Shea, Byun, \& Carr, 2001; Sakai, Ho, Shore, Risk, \& Price, 2005; Zane \& Kim,
1994), HED still occurs (Iwamoto, Takamatsu, \& Castellanos, 2012; Lee, Han, \& Gfroerer, 2013). Asian Americans are typically viewed as the "model minority" and as such, drinking issues may be wrongfully ignored (Yun \& Park, 2008). Differences in drinking rates have been connected to genetic or physical differences, and more recently, sociocultural factors have been shown to predict drinking patterns among Asian individuals as well (Hahm, Lahiff, \& Guterman, 2004). Cook, Mulia, and KarrikerJaffe (2012) reviewed data from the National Epidemiologic Study of Alcohol and Related Conditions and concluded that Asian immigrant drinking habits were correlated with country of origin per capita alcohol consumption. Individuals emigrating from countries with higher per capita consumption were more likely to be drinkers or drink more frequently. Among Asian Americans born in the United States, degree of endorsed

Correspondence: Danielle Ruderman, College of Social Work, 125 Stillman Hall, 1947 College Road, Columbus OH 43210. Telephone: 614-292-5572, Fax: 614-2926940, E-mail: Ruderman.5@osu.edu

Financial support: This project was supported by grant 1RC1AA018970-01 by NIH/NIAAA to C. Richard Hofstetter and intramural support by the Center for Behavioral and Epidemiology and Community Health, San Diego State University.

Keywords: Heavy episodic drinking, Korean immigrants, Chinese immigrants, social networks 
ethnic culture or use of ethnic language was associated with alcohol consumption (Cook et al., 2012). Asian Americans who utilized more ethnic language were less likely to be drinkers or to drink frequently.

Recent studies have detected a rise in alcohol-related problems among Asian Americans (Hahm et al., 2004; Iwamoto et al., 2012), which may be related to increased acculturation of younger generations (Cook et al., 2012; Grant et al., 2004; Hahm et al., 2004; Lum, Corliss, Mays, Cochran, \& Lui, 2009; Sakai et al., 2005; Yun \& Park, 2008). According to Hahm et al. (2004), Asian American adolescents with higher degrees of acculturation were more likely to drink. Furthermore, American-born persons were more likely to consume higher levels of alcohol (Lum et al., 2009). In a sample of 952 Asian Americans, degree of integration into American culture significantly influenced increased drinking (Cook et al., 2012).

Among studies that viewed within-group differences, Chinese individuals report the lowest substance use rates (Iwamoto et al., 2012; Lee et al., 2013; Wong, Klingle, \& Price, 2004). Individuals of Korean descent typically report the highest drinking rates among Asian subgroups (Lee et al., 2013; Lum et al., 2009; Parrish, 1995; Weatherspoon, Danko, \& Johnson, 1992). Compared to Asian non-drinkers, Asian individuals who reported higher levels of education, went to nightclubs, and did not attend weekly worship were more likely to drink alcohol (Chi, Lubben, \& Kitano, 1989). In general, few women reported drinking, and prevalence of HED was greater among Korean and Japanese men.

Past research focusing on Asian American substance use is limited and has been problematic due to insufficient sampling techniques. Samples are often composed of English-speaking high school or college students and may not be generalizable. Additionally, there are noticeable variations in substance use between Asian subgroups. Studies have clustered all Asian subgroups together, ignoring variability among cultures, and they often ignore level of acculturation, socioeconomic status, and religion (Varma \& Siris, 1996).

Based on the above, this study contributes to the existing literature by examining the differing impact of acculturation and social networks on subsets of Asian American populations. While past studies have determined differences in drinking rates among subpopulations, our study explores the impact of cultural differences between two nationalities and how these differences impact drinking rates. This study aims to determine whether there is a relationship between nationality (Chinese or Korean) and HED rates. We expect that Korean immigrants will report more HED occasions than Chinese immigrants. We also explore the impact of acculturation, predicting that acculturated persons are less likely than unacculturated persons to display HED. Additionally, this study focuses on how demographic factors or social network interactions may influence HED among these Asian immigrant populations. We hypothesize that networks composed of drinkers and/or problem drinkers and social interactions that encourage drinking will increase reported HED rates.

\section{Methods}

All study procedures were approved by the San Diego State University Human Subjects Committee.

\section{The Sample}

A dual frame sampling strategy was used to select firstgeneration Chinese and Korean adults (21 years and over) residing in three Los Angeles counties that are home to the majority of Chinese and Korean immigrants in southern California. Frame one was based on a random surname sample of persons residing in each county with listed telephone numbers (Lauderdale \& Kestenbaum, 2000; Myung, McDonnell, Kazinets, Seo, \& Moskowitz, 2010; Quan et al., 2006; Wong, Palaniappan, \& Lauderdale, 2010). Initial interviewing demonstrated that older persons (40 years or older), whose life styles were not representative of alcohol consumption among younger Chinese or Koreans, were more likely to have listed numbers.

Frame two included immigrants (40 years or younger) who were at greater risk for alcohol and other health concerns, and who are increasingly dependent on cell phones (Blyth, 2008; Couper, 2005; Lynn \& Kaminska, 2010; U.S. Department of Health and Human Services, 2011; 2012) and were not listed in available directories. Random digit dialing was not viable since groups were dispersed (Zhou, 2009) so that Chinese and Koreans each constitute relatively small proportions of a much larger populations within telephone exchanges. This frame involved nonprobability recruitment based on contacts with language schools (prominent in Asian communities), radio solicitations on Chinese and Korean stations, endorsements by ethnic organizations, attendance at ethnic fairs and celebrations, recreation facilities, educational institutions, solicitations outside Asian grocery stores in Asian shopping centers, and referrals from would-be survey participants older than the age stratum. Sampling details are available (Hofstetter et al., 2014). Interviews were completed with 1183 Chinese (773 in the probability sample and 410 in the non-probability frame) and 1531 Korean immigrants (861 in the probability sample and 670 in the non-probability frame). While non-probability procedures do not permit estimating population parameters precisely, the data do permit testing hypotheses and controlling statistically for the nature of sampling.

\section{Interviewing}

Structured phone interviews were conducted by two teams of professional bilingual interviewers, one fluent in Mandarin and English and the other in Korean and English, who were closely observed by bilingual supervisors during interviewing. Initial contact was made in Mandarin or Korean and the interview was conducted in the language of participants' preferences. Cooperation rates (percent of eligible participants contacted who completed interviews) 
were about $55.0 \%$ among Chinese and $65.0 \%$ among Koreans. Chinese and Korean translation of each item is available from the second author on request. A project 800 number was used to answer questions and also to permit potential respondents to contact project personnel. Interviews lasted for approximately 20 minutes.

\section{Questionnaires}

Questionnaires were designed and translated using a multistage procedure. Initially developed in English using measures that had been previously vetted, questions were translated into Mandarin and Korean by bilingual native speakers, reviewed by focus groups, back-translated, modified, and repeatedly put through these steps until optimal meanings of concepts were reached. English, Mandarin, and Korean translations are available on request from the authors.

\section{Measures}

Heavy Episodic Drinking. HED was measured by responses to the following: "During the last 12 months, how often did you have 5 or more drinks (for male respondents) or 4 or more drinks (for female respondents) containing any kind of alcohol within a two-hour period?" A drink was defined as a U.S. standard drink (12 ounces of beer, 5 ounces of wine, and 1.5 ounces of distilled spirits). This definition was provided to respondents. The U.S. standard drink size was utilized because all respondents resided in the United States. One or more heavy drinking episodes was coded as 1 and no HED episodes was coded as 0 , since the data had a strong right skew, with $64.7 \%$ reporting no HED.

Sampling Frame. Type of sampling was coded (1 for probability, 0 for non-probability).

Social Networks. Regular interaction was measured by responses to the following: "In this section, please think about your family, friends and other people you interact with here ... . think of all the people who live in America now, and that you see at least once a month. About how many are there?" Size of network was based on responses to the above and capped at 100 . The natural logarithm was computed to constrain skewness. Among these persons, interactions involving alcohol were measured by responses to the following:

a. "How many of them drink alcoholic beverages?" (Number that drink). Responses were transformed using the natural logarithm to constrain skewness.

b. Problem drinking of others (drinking behavior of social network): "So far as you know, how many of them have problems with drinking alcohol?" (dichotomized as yes $=1$, otherwise 0 ). Alcoholrelated problems were left to participant interpretation.

c. Encouragement: "How many of them encouraged you to drink alcoholic beverages?” (dichotomized as yes $=1$, otherwise 0 ).
Discouragement for drinking was also measured, but so few participants reported being discouraged that the variable was dropped from analysis.

Acculturation. Acculturation was measured by the participants' language preference during interviewing and coded 1 for English and 0 for Mandarin/Korean. Language use has been utilized to justify acculturation in past research (Ferdman, 1990; Finch, Frank, \& Vega, 2004).

Demographics. Demographics were measured by selfreport and included age (years), sex, U.S. citizenship, nationality, marital status, and years of formal education (capped at 20).

\section{Analysis}

Analyses were computed using Stata (version 13.1). Distributions of continuous variables were transformed when needed to constrain skewness. Hypotheses were tested by (logistic) regressing the occurrence of HED based on general and several social interaction variables after controlling for age, gender, education, marital status, acculturation (English language preference), ethnicity (Chinese/Korean), and subsample (probability/nonprobability). Missing data were imputed using the Stata MI procedure with a normal distribution of error assumption $(\mathrm{MVN})$ and 30 imputations based on the covariance structure of the data and random assignment of error (StataCorp, 2011). Highly collinear variables were excluded from multivariate analyses. Variables that were not found to be significant in the bivariate associations were still placed into the multivariate model in order to control for suppressed variables. A variable that may not be statistically significant in the bivariate analysis may become statistically significant in the multivariate analysis (Pandey \& Elliot, 2010).

\section{Results}

\section{Sample Characteristics (based on non-imputed data)}

According to Table 1, approximately half of the sample was recruited through probability procedures (56.2\% of the Korean sample and $65.3 \%$ of the Chinese sample). Most of the participants preferred to be interviewed in their native language (98.4\% of Koreans and $96.7 \%$ of Chinese). The sample was composed of about equal male and female participants of both nationalities, and $43.8 \%$ of Koreans and $65.4 \%$ of Chinese participant were U.S. citizens. About two thirds of the sample for both nationalities identified as married or cohabiting. Both Korean and Chinese participants reported completing approximately 15 years of education. Korean participants reported a mean age of 42.8 years, and Chinese participants reported a mean age of 43.6 years. Koreans also reported a mean social network size (transformed by logarithm) of 1.54 and mean others in networks who were drinkers of 1.06, in contrast to the Chinese participants, who reported 2.2 and 1.1, respectively. Koreans reported interacting with problem drinkers $3.2 \%$ of the time and being encouraged to drink 
Table 1

Sample characteristics by ethnic subgroup

\begin{tabular}{|c|c|c|c|c|c|c|c|}
\hline \multirow[b]{3}{*}{ Variable } & \multicolumn{3}{|c|}{ Koreans } & \multicolumn{3}{|c|}{ Chinese } & \multirow[b]{3}{*}{$p<$} \\
\hline & \multirow[b]{2}{*}{ Mean/\% } & \multicolumn{2}{|c|}{$\begin{array}{l}\text { 95\% confidence } \\
\text { interval }\end{array}$} & \multirow[b]{2}{*}{ Mean/\% } & \multicolumn{2}{|c|}{$\begin{array}{l}\text { 95\% confidence } \\
\text { interval }\end{array}$} & \\
\hline & & Lower & Upper & & Lower & Upper & \\
\hline HED_Dichotomized & $51.1 \%$ & $48.1 \%$ & $54.0 \%$ & $10.7 \%$ & $8.5 \%$ & $13.0 \%$ & .001 \\
\hline Probability sample & $56.2 \%$ & $53.7 \%$ & $58.7 \%$ & $65.3 \%$ & $62.6 \%$ & $68.0 \%$ & .001 \\
\hline Sex (males) & $53.2 \%$ & $50.7 \%$ & $55.7 \%$ & $50.8 \%$ & $48.0 \%$ & $53.7 \%$ & .001 \\
\hline U.S. citizen & $43.8 \%$ & $41.3 \%$ & $46.3 \%$ & $65.4 \%$ & $62.7 \%$ & $68.2 \%$ & .001 \\
\hline English language preference & $1.6 \%$ & $0.9 \%$ & $2.2 \%$ & $3.3 \%$ & $2.3 \%$ & $4.4 \%$ & .003 \\
\hline Married & $64.7 \%$ & $62.3 \%$ & $67.1 \%$ & $66.6 \%$ & $63.9 \%$ & $69.3 \%$ & .316 \\
\hline Education & 15.52 & 15.37 & 15.66 & 15.28 & 15.09 & 15.46 & .039 \\
\hline Age & 42.89 & 42.15 & 43.63 & 43.60 & 42.79 & 44.41 & .208 \\
\hline Size of network (ln) & 1.54 & 1.48 & 1.60 & 2.25 & 2.20 & 2.31 & .000 \\
\hline Network drinkers (ln) & 1.07 & 1.01 & 1.12 & 1.13 & 1.06 & 1.20 & .161 \\
\hline $\begin{array}{l}\text { Social models (Problem Drinkers } \\
\text { dichotomized) }\end{array}$ & $3.2 \%$ & $2.2 \%$ & $4.3 \%$ & $4.4 \%$ & $3.2 \%$ & $5.6 \%$ & .150 \\
\hline Support (Encourage Drinking Dichotomized) & $20.9 \%$ & $18.6 \%$ & $23.3 \%$ & $3.1 \%$ & $2.1 \%$ & $4.2 \%$ & .000 \\
\hline
\end{tabular}

Note: Numbers are means of percentages and 95\% confidence intervals for key study variables. Missing data have been deleted for each variable in calculations

Table 2

Bivariate and multivariate logistic regression of reported heavy episodic drinking on selected predictors (Imputation)

\begin{tabular}{|c|c|c|c|c|c|c|}
\hline \multirow[b]{3}{*}{ Predictor } & \multicolumn{2}{|c|}{ Bivariate } & \multicolumn{4}{|c|}{ Multivariate } \\
\hline & \multirow[b]{2}{*}{$O R$} & \multirow[b]{2}{*}{$p<$} & \multirow[b]{2}{*}{ OR } & \multirow[b]{2}{*}{$p<$} & \multicolumn{2}{|c|}{$95 \%$ CI } \\
\hline & & & & & Lower & Upper \\
\hline Probability sample & .385 & .001 & .758 & .022 & .599 & .960 \\
\hline Sex (males) & 2.932 & .001 & 3.047 & .000 & 2.392 & 3.879 \\
\hline U.S. citizen & .035 & .001 & .698 & .006 & .541 & .901 \\
\hline English language preference & 1.332 & .329 & 2.085 & .058 & .974 & 4.464 \\
\hline Married & .475 & .001 & .653 & .005 & .486 & .878 \\
\hline Chinese ethnicity & .108 & .001 & .088 & .000 & .064 & .122 \\
\hline Education & 1.059 & .001 & 1.008 & .699 & .964 & 1.055 \\
\hline Age & .954 & .001 & .969 & .000 & .956 & .982 \\
\hline Size of network (ln) & .733 & .001 & .870 & .105 & .735 & 1.029 \\
\hline Network drinkers (ln) & 1.184 & .001 & 1.379 & .002 & 1.127 & 1.687 \\
\hline Social models (Problem Drinkers Dichotomized) & 1.721 & .028 & 2.261 & .012 & 1.195 & 4.277 \\
\hline Social support (Encourage Drinking Dichotomized) & 4.338 & .001 & 1.783 & .002 & 1.244 & 2.556 \\
\hline Constant & & & 2.177 & .071 & .934 & 5.072 \\
\hline
\end{tabular}

CI, confidence interval; OR, odds ratio.

Note: Numbers in cells are bivariate and multivariate odds ratios, associated probabilities, and multivariate 95\% confidence intervals when one or more heavy episodic drinking occurrences are reported among first-generation Chinese and Korean immigrants in greater Los Angeles from 2010-2011. Missing values were imputed using the chained MI multiple imputation in Stata (version 13.1) with 30 imputations. Highly skewed continuous variables were dichotomized. The natural logarithm was computed among less skewed distributions (ln) to constrain skewness. 
$4.4 \%$ of the time; Chinese participants reported frequency of these occurrences at $4.4 \%$ and $3.1 \%$, respectively Koreans were significantly more likely than Chinese to report HED. Chinese were significantly more likely than Koreans to be a U.S. citizen, have a larger social network, and prefer English for the interview. Koreans were more likely than Chinese to be female, have slightly more education, and report networks that encouraged drinking. There were no statistically significant differences among Chinese and Korean participants for being married and for the number of problem drinkers in their social networks.

\section{Results from Multivariate Logistic Regression}

Table 2 reports bivariate and multivariate odds ratios, associated probabilities, and 95\% confidence intervals (CI) for the logistic regression of HED on selected predictors. Participants in the probability sample, U.S. citizens, married or cohabiting persons, Chinese, younger persons, and persons in larger networks were less likely to report HED in the bivariate analysis. Males, those more highly educated, and participants with networks composed of drinkers, problem drinkers, and those that encourage drinking were more likely to report HED. English language preference was not related to HED significantly $(p>.05)$ in the bivariate analysis.

In the multivariate analysis, men were 3.0 times more likely to report HED than women (odds ratio $[O R]=3.0$, 95\% CI $=2.4-3.9$ ). $\quad$ Participants with social networks composed of drinkers were 1.4 times more likely to report HED than those with networks that did not drink $(O R=1.4$, $95 \%$ CI $=1.1-1.7$ ). Furthermore, participants with social networks composed of problem drinkers were 2.3 times more likely to report HED than those without problem drinkers in their network $(O R=2.3,95 \% \mathrm{CI}=1.2-4.3)$. Participants who believed their network encouraged drinking were 1.8 times more likely to report HED than participants with networks that did not encourage drinking $(O R=1.8,95 \% \mathrm{CI}=1.2-2.6)$. Lastly, being in the probability sample, a U.S. citizen, married, Chinese, and of a younger age were protective of HED. English language preference, years of education, and size of one's social network did not contribute significantly to the model. The analysis was replicated using multiple imputation (Stata MI, StataCorp, 2011). The patterns of both coefficients and associated probabilities were similar to those reported above.

\section{Discussion}

This study supports past research (Lee et al., 2013) and illustrates that differences in drinking habits do exist among various Asian subgroups. As expected, Korean immigrants reported HED more frequently than Chinese immigrants. Furthermore, this study contributes to the literature about the impact of social relationships on drinking behaviors. There is a growing body of literature that depicts the importance of peer influences on the drinking behaviors of American drinkers (Borsari \& Carey, 2006; DeMartini, Prince, \& Carey, 2013). For instance, a larger social network of heavy drinkers is associated with an increase in HED for its members (Delucchi, Matzger, \& Weisner, 2008). If one's friends are supportive of drinking, heavy drinking behavior is more likely to occur. Social variables have been shown to be influencing factors in drinking behaviors among Asian immigrant populations as well (Hahm et al., 2004). Our study further supports the relationship between peers and risky behavior. It is feasible that the participants in this study were swayed by interactions with people in their social network (though future research could explore this more specifically). When the number of friends who also consumed alcohol increased, the likelihood of HED increased. HED was also influenced by encouragement. If participants felt their friends encouraged their drinking, HED in the past year was more likely to occur.

The logistic regression model also demonstrated that immigrants who became U.S. citizens were less likely to report past-year HED. Additionally, acculturation was not significant in the model even though this is a factor in past research (Cook et al., 2012; Lum et al., 2009). It is somewhat atypical that heavy drinking increased with age; however, this could be evidence that behaviors change with more time spent in the United States. Marriage or cohabiting was protective of heavy drinking. Marital status indicates, however crudely, a key social linkage that may provide support, aid, and control and is therefore related to any number of health variables. Immigrants are under considerable stress and other family members may be an integral provider of key resources.

This study has several limitations, including a reliance on self-report data, which may have some biases. Asian Americans may feel hesitant to admit to alcohol-use issues due to social pressures. This study attempted to mitigate this problem by allowing each participant to choose which language they felt most comfortable using. The sampling method may have been problematic. Only about half of those sampled agreed to respond to the survey items. Those sampled by probability methods were more likely to report HED and the sampling method was a significant factor in the logistic regression model. It is unclear why participants in the probability sample were less likely to report HED. Nonetheless, this large sample of firstgeneration immigrants was recruited from one of several major regions of Chinese and Korean settlement in the United States. Furthermore, the use of participants' language as a measure of acculturation is potentially problematic. This method was used in previous studies (Hofstetter et al., 2004; Hofstetter et al., 2014); however, participants may adopt U.S. customs or be impacted by the culture in other ways. Language is only one example. Past HED was assessed over a 12-month period instead of the typical shorter duration of 30 days. This may impact participant recollection; nonetheless, we chose this timeframe in order to have a large enough prevalence to view relationships in the data. We allowed participants to interpret acquaintances' problem drinking in their social network. Participants may have defined alcohol-related problems differently. Additionally, the data are crosssectional and do not represent changes in drinking habits 
over time. Future research could follow participants longitudinally to view the relationship between time spent in the United States, acculturation, and alcohol use.

Recent research has illustrated an increase in alcoholrelated problems in Asian immigrant populations (Cook et al., 2012; Lum et al., 2009). This is especially concerning given the rapid growth of this population in the United States. Korean and Chinese immigrants may be drinking less than their Caucasian counterparts; however, HED among these populations is still of concern and warrants prevention. This study illustrates that alcohol-use problems in Asian immigrants is not homogenous, and effective prevention, intervention, and treatment should take into account the varying drinking rates of Asian subgroups. Future research on problematic outcomes of drinking and potential barriers to services and treatment among Asian subgroups is warranted. This research provides an extension to the literature that Asian Americans are impacted by their social networks and provides an important foundation to address this risk factor further.

\section{References}

Akutsu, P. D., Sue, S., Zane, N. W. S., \& Nakamura, C. Y. (1989). Ethnic differences in alcohol consumption among Asian and Caucasians in the United States: An investigation of cultural and physiological factors. Journal of Studies on Alcohol, 50, 261-267. doi:10.15288/jsa.1989.50.261

Blyth, B. (2008). Mixed mode: The only fitness' regime? International Journal of Market Research, 50(2), 241266.

Borsari, B., \& Carey, K. B. (2006). How the quality of peer relationships influences college alcohol use. Drug and Alcohol Review, 25, 361-370. doi:10.1080/09595230600741339

Centers for Disease Control and Prevention. (2013). Binge drinking - United States, 2011. Morbidity and Mortality Weekly Report, 62(3), 77-80.

Chi, I., Lubben, J. E., \& Kitano, H. H. (1989). Differences in drinking behavior among three Asian-American groups. Journal of Studies on Alcohol, 50(1), 15-23.

Cook, W. K., Mulia, N., \& Karriker-Jaffe, K. (2012). Ethnic drinking cultures and alcohol use among Asian American adults: Findings from a national survey. Alcohol and Alcoholism, 47(3), 340-348. doi:10.1093/alcalc/ags017

Couper, M. P. (2005). Technology trends in survey data collection. Social Science Computer Review, 23(4), 486-501. doi:10.1177/0894439305278972

Delucchi, K. L., Matzger, H., \& Weisner, C. (2008). Alcohol in emerging adulthood: 7 year study of problem and dependent drinkers. Addictive Behaviors, 33, 134-142. doi:10.1016/j.addbeh.2007.04.027

DeMartini, K. S., Prince, M. A., \& Carey, K. B. (2013). Identification of trajectories of social network composition change and the relationship to alcohol consumption and norms. Drug and Alcohol Dependence, 132, 309-315. doi:10.1016/j.drugalcdep.2013.02.020
Ferdman, B. (1990). Literacy and cultural identity. Harvard Educational Review, 60(2), 181-205.

Finch, B. K., Frank, R., \& Vega, W. A. (2004). Acculturation and acculturation stress: A socialepidemiological approach to Mexican migrant farmworkers' health. International Migration Review, 236-262. doi:10.1111/j.1747-7379.2004.tb00195.x

Grant, B. F., Dawson, D. A., Stinson, F. S., Chou, S. P., Dufour, M. C., \& Pickering, R. P. (2004). The 12month prevalence and trends in DSM-IV alcohol abuse and dependence: United States, 1991-1992 and 2001-2002. Drug and Alcohol Dependence, 74, 233234. doi:10.1016/j.drugalcdep.2004.02.004

Hahm, H. C., Lahiff, M., \& Guterman, N. B. (2004). Asian American adolescents' acculturation, binge drinking, and alcohol and tobacco-using peers. Journal of Community Psychology, 32(3), 295-308. doi:10.1002/jcop.20002

Hoeffel, E. M., Rastogi, S., Kim, M. O., \& Shahid, H. (2012). The Asian Population 2010. Census 2010 Brief. U.S. Census Bureau. Retrieved from https://www.census.gov/prod/cen2010/briefs/c2010br11.pdf.

Hofstetter, C. R., Clapp, J. D., Allem, J. P., Hughes, S. C., Irvin, V., Kang, S., \& Hovell, M. F. (2014). Social networks and alcohol consumption among first generation Chinese and Korean immigrants in the Los Angeles metropolitan area. International Journal of Alcohol Studies, 3(4), 245-255. doi:10.7895/ijadr.v3i4.188

Hofstetter, C. R., Hovell, M. F., Lee, J., Zakarian, J., Park, H., Paik, H. Y., \& Irvin, V. (2004). Tobacco use and acculturation among Californians of Korean descent: A behavioral epidemiological analysis. Nicotine \& Tobacco Research, 6(3), 481-489. doi:10.1080/14622200410001696646

Iwamoto, D., Takamatsu, S., \& Castellanos, J. (2012). Binge drinking and alcohol-related problems among U.S.-born Asian Americans. Cultural Diversity and Ethnic Minority Psychology, 18(3), 219-227. doi:10.1037/a0028422

Lauderdale, D. S., \& Kestenbaum, B. (2000). Asian American ethnic identification by surname. Population Research and Policy Review, 19(3), 283300.

Lee, H. K., Han, B., \& Gfroerer, J. C. (2013). Differences in the prevalence rates and correlates of alcohol use and binge alcohol use among five Asian American subpopulations. Addictive Behaviors, 38(3), 18161823. doi:10.1016/j.addbeh.2012.11.001

Luczak, S. E., Wall, T. L., Shea, S. H., Byun, S. M., \& Carr, L. G. (2001). Binge drinking in Chinese, Korean, and White college students: Genetic and ethnic group differences. Psychology of Addictive Behaviors, 15(4), 306-309. doi:10.1037//0893-164x.15.4.306

Lum, C., Corliss, H. I., Mays, V. M., Cochran, S. D., \& Lui, C. K. (2009). Differences in the drinking behaviors of Chinese, Filipino, Korean, and Vietnamese college students. Journal of Studies on Alcohol and Drugs, 70, 568-574. doi:10.15288/jsad.2009.70.568 
Lynn, P., \& Kaminska, O. (2010). The impact of mobile phones on survey measurement error. Retrieved from: https://www.iser.essex.ac.uk/files/iser_working_paper s/2011-07.pdf

Myung, S. K., McDonnell, D. D., Kazinets, G., Seo, H. G., \& Moskowitz, J. M. (2010). Relationships between household smoking restrictions and intention to quit smoking among Korean American male smokers in California. Journal of Korean Medical Science, 25(2), 245-250. doi:10.3346/jkms.2010.25.2.245

Pandey, S., \& Elliott, W. (2010). Suppressor variables in social work research: Ways to identify in multiple regression models. Journal of the Society for Social Work and Research, 1(1), 28-40.

Parrish, K. M. (1995). Alcohol abuse prevention research in Asian American and Pacific Islander communities. In P. A. Langton, L. G. Epstein, \& M. A. Orlandi (Eds.), Challenging of participatory research: Preventing alcohol-related problems in ethnic communities (pp. 411-428). Center for Substance Abuse Prevention Cultural Competence Series 3. Rockville, MD, United States: Center for Substance Abuse Prevention.

Quan, H., Wang, F., Schopflocher, D., Norris, C., Galbraith, P. D., Faris, P., . . G Ghali, W. A. (2006). Development and validation of a surname list to define Chinese ethnicity. Medical Care, 328-333. doi:10.1097/01.mlr.0000204010.81331.a9

Sakai, J. T., Ho, P. M., Shore, J. H., Risk, N. K., \& Price, R. K. (2005). Asians in the United States: Substance dependence and use of substance-dependence treatment. Journal of Substance Abuse Treatment, 29, 75-84. doi:10.1016/j.jsat.2005.04.002

StataCorp. (2011). Stata: Release 12 multiple-imputation reference manual (Vol. Release 12). College Station, TX, United States: StataCorp LP.

Substance Abuse and Mental Health Services Administration. (2010). Results from the 2010 national survey on drug use and health: Summary of national findings. Retrieved from: http://www.oas.samhsa.gov/NSDUH/2k10NSDUH/2k 10Results.htm

U.S. Department of Health and Human Services, Centers for Disease Control and Prevention, National Center for Health Statistics. (2011). Wireless substitution: State-level estimates from the National Health Interview Survey, January 2007-June 2010. Retrieved from: http://beta.newsleader.com/assets/pdf/DO173321420.PDF

U.S. Department of Health and Human Services, Centers for Disease Control and Prevention, National Center for Health Statistics. (2012). Wireless substitution: State-level estimates from the National Health Interview Survey, 2010-2011. Retrieved from: http://198.246.124.22/nchs/data/nhsr /nhsr061.pdf

U.S. Census Bureau. (2012). The Asian population: 2010. Retrieved from: http://www.census.gov/prod/ cen2010/briefs/c2010br-11.pdf

Varma, S. C., \& Siris, S. G. (1996). Alcohol abuse in Asian Americans. American Journal on Addictions, 5, 136143. doi:10.1111/j.1521-0391.1996.tb00296.x

Weatherspoon, A. J., Danko, G. P., \& Johnson, R. C. (1992). Alcohol consumption and use norms among
Chinese Americans and Korean Americans. Journal of Studies on Alcohol, 55, 203-206. doi:10.15288/jsa.1994.55.203

Wong, E. C., Palaniappan, L. P., \& Lauderdale, D. S. (2010). Using name lists to infer Asian racial/ethnic subgroups in the healthcare setting. Medical Care, 48(6), 540-546. doi:10.1097/mlr.0b013e3181d559e9

Wong, M. M., Klingle, R. S., \& Price, R. K. (2004). Alcohol, tobacco, and other drug use among Asian American and Pacific Islander Adolescents in California and Hawaii. Addictive Behaviors, 29, 127141. doi:10.1016/s0306-4603(03)00079-0

Yun, S. H., \& Park, W. (2008). Clinical characteristics of alcohol drinking and acculturation issues faced by Korean immigrants in the United States. Journal of Social Work Practice in Addictions, 8(1), 3-20. doi:10.1080/15332560802108597

Zane, N. W., \& Kim, J. C. (1994). Substance use and abuse. In N. W. Zane, D. T. Takeuchi, \& K. N. J. Young (Eds.), Confronting critical health issues of Asian and Pacific Islander Americans (pp. 316-346). Thousand Oaks, CA, United States: Sage Publications.

Zhou, M. (2009). Contemporary Chinese America: Immigration, ethnicity, and community transformation. Philadelphia, PA, United States: Temple University Press. 